This item was submitted to Loughborough's Research Repository by the author.

Items in Figshare are protected by copyright, with all rights reserved, unless otherwise indicated.

\title{
Integrated design of dynamic controller with fault diagnosis and tolerance
}

PLEASE CITE THE PUBLISHED VERSION

PUBLISHER

(C) IEEE

VERSION

VoR (Version of Record)

LICENCE

CC BY-NC-ND 4.0

REPOSITORY RECORD

Li, Zhenhai, Argyrios C. Zolotas, I.M. Jaimoukha, and K.M. Grigoriadis. 2019. "Integrated Design of Dynamic Controller with Fault Diagnosis and Tolerance". figshare. https://hdl.handle.net/2134/5340. 
This item was submitted to Loughborough's Institutional Repository (https://dspace.lboro.ac.uk/) by the author and is made available under the following Creative Commons Licence conditions.

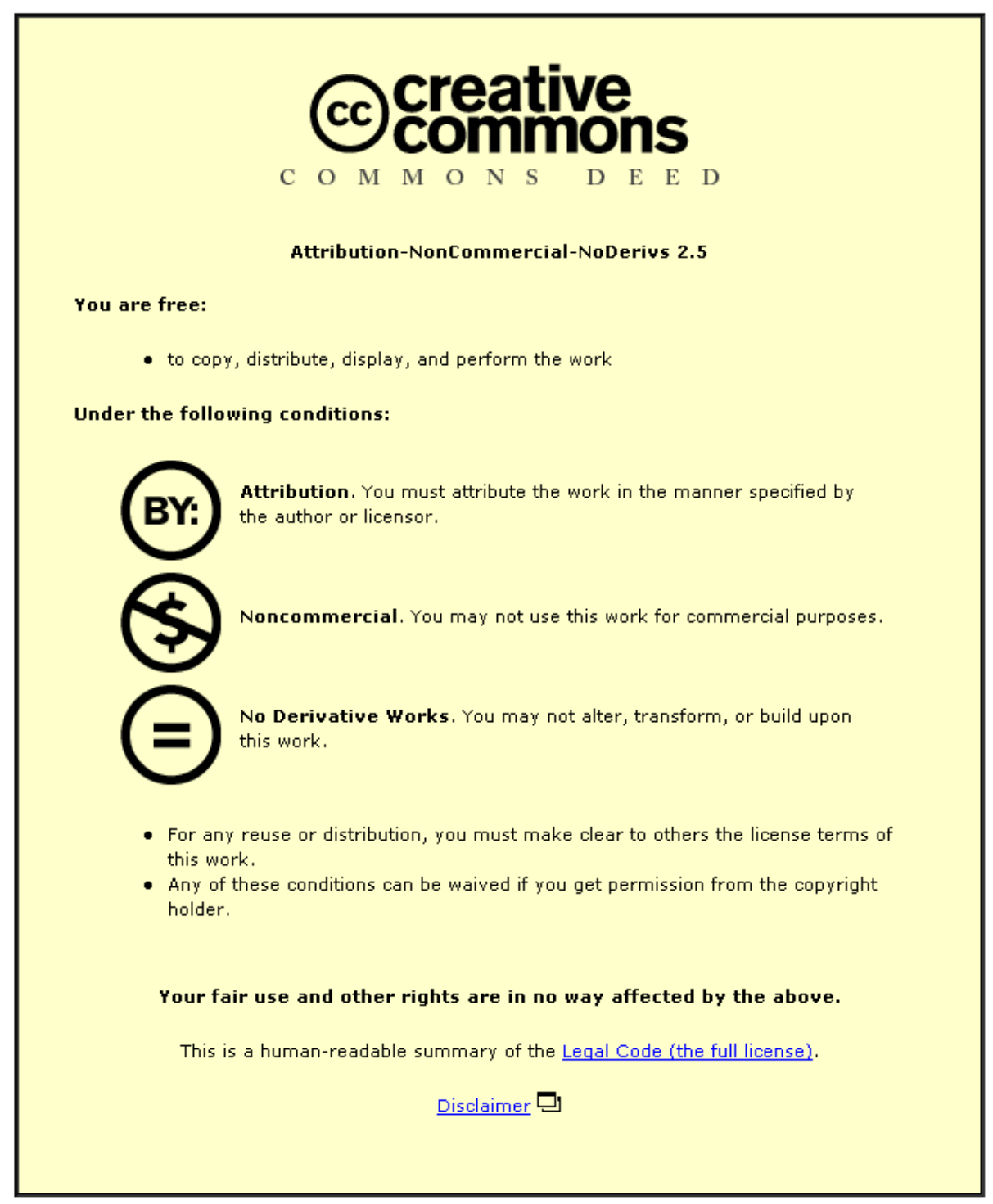

For the full text of this licence, please go to: http://creativecommons.org/licenses/by-nc-nd/2.5/ 


\title{
Integrated Design of Dynamic Controller with Fault Diagnosis and Tolerance
}

\author{
Zhenhai Li, Argyrios Zolotas, Imad Jaimoukha and Karolos Grigoriadis
}

\begin{abstract}
Fault detection capability tends to become an integral part of control system design procedures for practical engineering systems. It is thus desirable fault diagnosis/tolerance functions to also be included in the controller design. In this context, we develop a generic observer-based feedback controller where the observer-part can also generate a residual signal for fault detection purposes. The design objectives is a mixture of $\mathcal{H}_{\infty}$ control and $\mathcal{H}_{\infty}$ fault detection and isolation. This multi-objective optimization problem is then formulated using Bilinear Matrix Inequalities (BMI) and a sub-optimal solution is achieved via transformation to Linear Matrix Inequalities (LMI). The developed approach and algorithm are verified in study of an application to a railway suspension system of ride quality maintenance.
\end{abstract}

\section{INTRODUCTION}

Modern control and monitoring systems involving a significant number of actuators and sensors prone to failure (in particular referring to the latter) are becoming rather complex and demanding in terms of maintenance. In a variety of practical engineering systems (aerospace, process, railway, and miscellanea electro-mechanical related systems) and for a given requirement, the practical engineering issue in the context of this paper is whether a particular level of fault diagnosis/tolerance can be integrated into the control design.

Observer-based fault detection (FD) schemes, one of the model-based fault detection related schemes, use dedicated state/output observer (or banks of such observers) to generate residual signals thus providing fault signatures will be encountered [1], [2], [3], [4]. The observer effectively cancels the process dynamics and is sensitive only to disturbances and faults. The filter design objective is then to reduce the sensitivity to disturbances while maintaining a given level of sensitivity to faults. Moreover, in observer-based controllers the choice of the number, location and type of sensor information (subject to sufficient set of actuator inputs for the addressed application) might have a significant impact on the overall performance, the complexity and the cost of the system. Most of the previous observer-based FD schemes operate in open-loop structures, namely, feedback control objectives are not involved in FD filter design. However, there are elements of work on integration of control and

This research was supported under the EPSRC project Grant Ref. EP/D063965/1 and BAE Systems.

Zhenhai $\mathrm{Li}$ and Argyrios Zolotas are with Department of Electronic and Electrical Engineering, Loughborough University, UK a.c.zolotaselboro.ac.uk

Imad Jaimoukha is with the Department of Electrical and Electronic Engineering, Imperial College London, UK i.jaimouka@imperial.ac.uk

Karolos Grigoriadis is with the Department of Mechanical Engineering, University of Houston, USA karolos@uh. edu fault detection that have appeared in the literature although rather different compared to the one proposed in this paper, i.e. in [5], [6] an integrated approach was presented for fault tolerant control mainly via Youla parametrizations while addressed issues with uncertainty on the separation of the control and fault detection and raised a series of interesting issues.

In this paper we investigate a framework which incorporates both a feedback controller design and fault detection design into a generic dynamic observer. Hence, fault detectability and fault tolerance can be considered as well as closed-loop control performance in the initial stage of a control design. Multi-objective formulation via Matrix Inequalities (MI) can provide the optimal mixed $\mathcal{H}_{\infty}$ performance for control and simple passive fault tolerance, will be encountered and $\mathcal{H}_{\infty}$ performance for fault detection. The $\mathcal{H}_{\infty}$ performance is employed to cover a worst case control purpose and guarantee fault indication to be maximally insensitive to disturbances for a given minimum level of sensitivity to faults. Also, an equivalent performance index for optimisation is given to apply appropriate Linear MI algorithms.

The paper is organised as follows: Section II gives a performance formulation of the control problem with consideration of fault detectability via dynamic observers. Section III transforms the problem into a state space framework via BMI and subsequent LMI solutions via variable transformations. An illustrative example addressing ride quality of a railway suspension system is given in Section IV while concluding remarks are made and future research directions are indicated in Section V.

The notation we use is mostly standard and is summarized next for convenience.The set of real (complex) $n \times m$ matrices is denoted by $\mathcal{R}^{n \times m}\left(\mathcal{C}^{n \times m}\right)$. For $A \in \mathcal{C}^{n \times m}$ we use the notation $A^{T}$ and $A^{\prime}$ to denote the transpose and complex conjugate transpose, respectively. For $A=A^{\prime} \in$ $\mathcal{C}^{n \times n}, A \geq 0$ denotes that $A$ is positive semidefinite (that is, all the eigenvalues of $A$ are greater than or equal to zero). For $A=A^{\prime} \in \mathcal{C}^{n \times n}, \bar{\lambda}(A)$ denotes the largest and $\underline{\lambda}(A)$ the smallest eigenvalue of $A$, respectively. For $A \in \mathcal{C}^{n \times m}, \bar{\sigma}(A)$ denotes the largest, and $\underline{\sigma}(A)$ the smallest, singular values of $A$, respectively. The $n \times n$ identity matrix is denoted as $I_{n}$ and the $n \times m$ null matrix is denoted as $0_{n, m}$ with the subscripts occasionally dropped if they can be inferred from context.

$\mathcal{R}(s)^{m \times p}$ denotes the set of all $m \times p$ real rational matrix functions of $s . \mathcal{L}_{\infty}^{m \times p}$ denotes the space of $m \times p$ matrix functions with entries bounded on the extended imaginary 
axis $j \mathcal{R}_{e}$. The subspace $\mathcal{H}_{\infty}^{m \times p} \subset \mathcal{L}_{\infty}^{m \times p}$ denotes matrix functions analytic in the closed right-half of the complex plane. A prefix $\mathcal{R}$ denotes a real rational function, so that $\mathcal{R} \mathcal{H}_{\infty}^{m \times p}$ denotes the set of all $m \times p$ stable real rational matrix functions of $s$. For $G(s) \in \mathcal{R} \mathcal{H}_{\infty}^{m \times p}$ we define

$$
\|G\|_{\infty}=\sup _{\omega \in \mathcal{R}} \bar{\sigma}(G(j \omega)), \quad\|G\|_{-}=\inf _{\omega \in \mathcal{R}} \underline{\sigma}(G(j \omega)) .
$$

For $G(s) \in \mathcal{R} \mathcal{L}_{\infty}^{m \times p}$, we define $G^{\sim}(s)=G(-s)^{T}$ to be the para-Hermitian complex conjugate transpose of $G(s)$.

\section{PROBLEM FORMULATION}

In our previous work the problem of output selection for fault tolerance with constraint disturbance rejection was addressed [7], however in this paper we propose a closedloop framework integrating control and observer capabilities with the observer error dynamics utilized in the form of a residual generation for the purposes of fault detection, isolation and other post-fault configuration, as depicted in Figure 1.

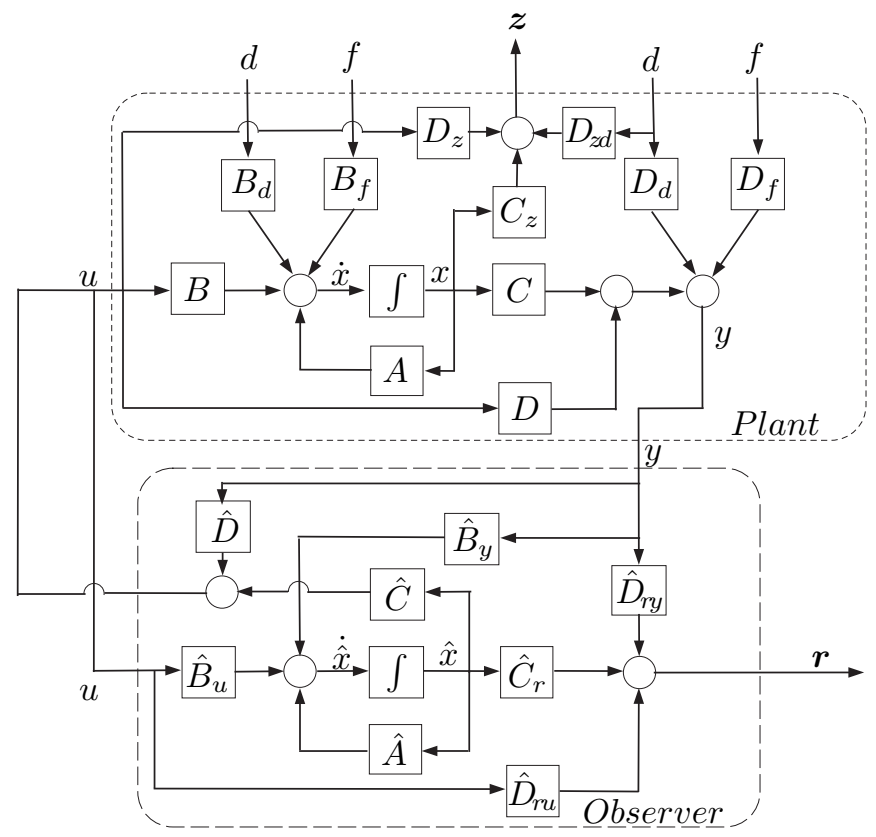

Fig. 1. Generalized regulator with dynamic observer-based controller and fault detection filter.

Note that $d(s)$ characterise any exogenous inputs entering the system, $u(s)$ is the fixed set of control inputs, $y(s)$ the measurements (their number is varying depending on the scenario) and the $z(s)$ includes the regulated outputs (w.r.t. $\infty$-norm or 2 -norm). Here, $\infty$-norm is considered as our control objectives.

Actuator faults and sensor faults can have channels to affect state dynamics and measured outputs directly, particularly in a feedback control system [8], [9]. Here, consider a linear time invariant (LTI) dynamic system subject to disturbances, actuator and sensor faults modeled as

$$
\begin{aligned}
\dot{x}(t) & =A x(t)+B_{d} d(t)+B u(t)+B_{f} f(t), \\
z(t) & =C_{z} x(t)+D_{z d} d(t)+D_{z} u(t), \\
y(t) & =C x(t)+D_{d} d(t)+D u(t)+D_{f} f(t),
\end{aligned}
$$

where $x(t) \in \mathcal{R}^{n_{p}}, u(t) \in \mathcal{R}^{n_{p u}}$ and $y(t) \in \mathcal{R}^{n_{p y}}$ are the state, input and output vectors, respectively and $w(t) \in \mathcal{R}^{n_{w}}$ is the disturbance vector. The energy of the output signal $z(t) \in \mathcal{R}^{n_{p 1}}$ is bounded for finite energy input signals by regulating the $\mathcal{H}_{\infty}$ norm of the system input-output gain (robustness metric). Here, $B_{d} \in \mathcal{R}^{n_{p} \times n_{w}}$, $D_{z d} \in \mathcal{R}^{n_{p 1} \times n_{w}}$ and $D_{d} \in \mathcal{R}^{n_{p y} \times n_{w}}$ are the corresponding disturbance distribution matrices, and $B \in \mathcal{R}^{n_{p} \times n_{p u}}, D_{z} \in$ $\mathcal{R}^{n_{p 1} \times n_{p u}}$ and $D \in \mathcal{R}^{n_{p y} \times n_{p u}}$ are the corresponding control distribution matrices, respectively. Similarly, $B_{f}$ and $D_{f}$ are known and well-defined fault channel distribution matrices with appropriate dimensions. Without loss of generality, we can assume $D=0$ (which is a generic assumption in $\mathcal{H}_{\infty}$ control problem).

\section{A. Generic Dynamic Observer}

We propose a generic dynamic observer which incorporates the controller design and fault detection design into one single observer/filter, both in closed-loop.

The observer has a dynamic model as

$$
\begin{aligned}
\dot{\hat{x}}(t) & =\hat{A} \hat{x}(t)+\hat{B}_{u} u(t)+\hat{B}_{y} y(t), \\
u(t) & =\hat{C} \hat{x}(t)+\hat{D} y(t), \\
r(t) & =\hat{C}_{r} \hat{x}(t)+\hat{D}_{r u} u(t)+\hat{D}_{r y} y(t),
\end{aligned}
$$

where $r(t) \in \mathcal{R}^{n_{f} \times n_{p y}}$ is the so-called residual signal representing the inconsistency between the system variables and the model. $\hat{A}, \hat{B}_{u}, \hat{B}_{y}, \hat{C}, \hat{D}, \hat{C}_{r}, \hat{D}_{r u}$ and $\hat{D}_{r y}$ are constant observer gain matrices to be determined with appropriate dimensions. We emphasize the introduction of the residual in the structure of our observer-based controller.

By defining an augmented state $x_{a}(t)=\left[\begin{array}{l}x(t) \\ \hat{x}(t)\end{array}\right]$, it can be easily shown that the dynamics of the closed-loop system are given by

$$
\begin{aligned}
\dot{x_{a}} & =A_{c l} x_{a}+B_{c l} d+F_{c l} f, \\
z & =C_{z c l} x_{a}+D_{z c l} d+F_{z c l} f, \\
r & =C_{r c l} x_{a}+D_{r c l} d+F_{r c l} f,
\end{aligned}
$$

where

$$
\begin{aligned}
& A_{c l}=\left[\begin{array}{cc}
A+B \hat{D} C & B \hat{C} \\
\hat{B}_{u} \hat{D} C+\hat{B}_{y} C & \hat{A}+\hat{B}_{u} \hat{C}
\end{array}\right], \\
& B_{c l}=\left[\begin{array}{c}
B_{d}+B \hat{D} D_{d} \\
\hat{B}_{u} \hat{D} D_{d}+\hat{B}_{y} D_{d}
\end{array}\right], F_{c l}=\left[\begin{array}{c}
B_{f}+B \hat{D} D_{f} \\
\hat{B}_{u} \hat{D} D_{f}+\hat{B}_{y} D_{f}
\end{array}\right] \text {, } \\
& C_{z c l}=\left[\begin{array}{ll}
C_{z}+\hat{D} C & D_{z} \hat{C}
\end{array}\right], D_{z c l}=D_{z d}+D_{z} \hat{D} D_{d} \text {, } \\
& F_{z c l}=D_{z} \hat{D} D_{f}, \\
& C_{r c l}=\left[\begin{array}{ll}
\hat{D}_{r u} \hat{D} C+\hat{D}_{r y} C & \hat{C}_{r}+\hat{D}_{r u} \hat{C}
\end{array}\right], \\
& D_{r c l}=\hat{D}_{r u} \hat{D} D_{d}+\hat{D}_{r y} D_{d} \text {, } \\
& F_{r c l}=\hat{D}_{r u} \hat{D} D_{f}+\hat{D}_{r y} D_{f} \text {. }
\end{aligned}
$$


Applying Laplace transforms, we can re-arrange the system in the following form

$$
\left.\begin{array}{ccc} 
& n_{d} & n_{f} \\
n_{p 1} & {\left[T_{z d}(s)\right.} & T_{z f}(s)
\end{array}\right] \quad \stackrel{s}{=}\left[\begin{array}{c|c|cc}
A_{c l} & B_{c l} & F_{c l} \\
\hline C_{z c l} & D_{z c l} & F_{z c l}
\end{array}\right]
$$

and

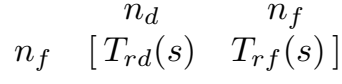

$$
\begin{aligned}
& \stackrel{s}{=}\left[\begin{array}{c|cc}
A_{c l} & B_{c l} & F_{c l} \\
\hline C_{r c l} & D_{r c l} & F_{r c l}
\end{array}\right]
\end{aligned}
$$

Here, $T_{z d}$ is the transfer function from $d$ to $z, T_{r d}$ is the transfer function from $d$ to the residual $r$ and $T_{r f}$ is the transfer function from $f$ to the residual $r$, respectively.

\section{B. Performance Index Including Residual}

Our objective is then to explore the channels by which a certain level of system performance is maintained and faults have most effect on the residual signal to indicate potential faults. Then the observer-based controller can also be utilised for post-fault configuration and fault tolerance.

We can integrate the above multiple objectives of control, fault diagnosis and tolerance into a single performance index by redefining the error dynamics. We set $e=r-f$ which results that

$$
e(s)=T_{e d}(s) d(s)+T_{e f}(s) f(s),
$$

where

$$
T_{e d}=T_{r d} \stackrel{s}{=}\left[\begin{array}{c|c}
A_{c l} & B_{c l} \\
\hline C_{r c l} & D_{r c l}
\end{array}\right]
$$

and

$$
T_{e f}=\stackrel{s}{=}\left[\begin{array}{c|c}
A_{c l} & F_{c l} \\
\hline C_{r c l} & F_{r c l}-I
\end{array}\right] .
$$

The problem is modified as following: find the observer/controller such that

$$
\rho=\gamma_{o}:=\inf \left\|T_{i n f}\right\|_{\infty}
$$

is obtained, where $T_{i n f}:=\left[\begin{array}{cc}T_{z d} & T_{z f} \\ T_{e d} & T_{e f}\end{array}\right]$ has the state space realization as

$T_{i n f} \stackrel{s}{=}\left[\begin{array}{c|c}A_{i n f} & B_{i n f} \\ \hline C_{\text {inf }} & D_{\text {inf }}\end{array}\right] \stackrel{s}{=}\left[\begin{array}{c|cc}A_{c l} & B_{c l} & F_{c l} \\ \hline C_{z c l} & D_{z c l} & F_{z c l} \\ C_{r c l} & D_{r c l} & F_{r c l}-I\end{array}\right]$.

\section{MATRIX INEQUALITY FORMULATION}

Optimizations problems in the area of robust control have been well studied via generalized LMI treatment [10]. However, in our case the multi-objective problem is more complex since designing the controller and fault detection filter is coupled which introduces nonlinear terms in the Matrix Inequality representation.

Let us develop the suboptimal solution of observer-based filter for control performance first. By virtue of the Bounded
Real Lemma [11], $A_{c l}$ is stable and $\left\|T_{z d}\right\|_{\infty}<\gamma_{1}$ if and only if there exists a symmetric $P$ with $P>0$ and

$$
T_{z c l}:=\left[\begin{array}{ccc}
P A_{c l}+A_{c l}^{T} P & \star & \star \\
B_{c l}^{T} P & -\gamma_{1} I & \star \\
C_{z c l} & D_{z c l} & -\gamma_{1} I
\end{array}\right]<0
$$

where $\star$ denotes terms readily inferred from symmetry.

However, the matrix inequality in (10) cannot be solved directly using convex optimization algorithms since nonlinear terms in the matrix inequalities will be encountered [10]. Boldface letters are used to indicate variables.

\section{A. Solution to the Generic Dynamic Observer Problem}

By virtue of the Bounded Real Lemma [11], $A_{\text {inf }}$ is stable and $\left\|T_{i n f}\right\|_{\infty}<\gamma$ if and only if there exists a symmetric $P$ with $P>0$ and

$$
T_{m i}:=\left[\begin{array}{ccc}
P A_{i n f}+A_{i n f}^{T} P & \star & \star \\
B_{i n f}^{T} P & -\gamma I & \star \\
C_{i n f} & D_{i n f} & -\gamma I
\end{array}\right]<0
$$

where $\star$ denotes terms readily inferred from symmetry.

The following result using the well-known technique change of variables inspired by [10], provides a bilinear formulation of the optimization problem of (8), which is solvable analytically via the LMI toolbox. Again boldface letters are used to indicate variables.

Lemma 3.1: Let all variables be defined as above, then a stabilizing dynamic controller exists such that $\left\|T_{i n f}\right\|_{\infty}<\gamma$ is achieved if there exists $X, Y, \tilde{A}, \tilde{B}_{y}, \tilde{C}, \tilde{C}_{r}, \hat{D}$ and $\bar{D}_{r y}$ such that (12) is true.

Then, the stabilizing dynamic controller is given by

$$
\begin{aligned}
\bar{C}_{r}= & \left(\tilde{C}_{r}-\bar{D}_{r y} C X\right) M^{-T} \\
\hat{C}= & (\tilde{C}-\hat{D} C X) M^{-T} \\
\bar{B}_{y}= & N^{-1}\left(\tilde{B}_{y}-Y B \hat{D}\right) \\
\bar{A}= & N^{-1}(\tilde{A}-Y A X-Y B \hat{D} C X \\
& \left.-N \bar{B}_{y} C X-Y B \hat{C} M^{T}\right) M^{-T}, \\
\hat{A}= & \bar{A}-\hat{B}_{u} \hat{C} \\
\hat{B}_{y}= & \bar{B}_{y}-\hat{B}_{u} \hat{D} \\
\hat{C}_{r}= & \bar{C}_{r}-\hat{D}_{r u} \hat{C} \\
\hat{D}_{r y}= & \bar{D}_{r y}-\hat{D}_{r u} \hat{D}
\end{aligned}
$$

where $\hat{B}_{u}$ and $\hat{D_{r u}}$ are arbitrary matrices with appropriate dimension, and square and nonsingular $M$ and $N$ should be (9) chosen such that

$$
M N^{T}=I-X Y .
$$

Proof We decompose the Lyapunov Matrix $P$ in (11) as the following

$$
P=\left[\begin{array}{cc}
Y & N \\
N^{T} & \hat{Y}
\end{array}\right], P^{-1}=\left[\begin{array}{cc}
X & M \\
M^{T} & \hat{X}
\end{array}\right],
$$

where $X, Y, \hat{X}, \hat{Y} \in \mathcal{R}^{n \times n}$ are symmetric and nonsingular. Let

$$
Q=\left[\begin{array}{cc}
X & I \\
M^{T} & 0
\end{array}\right], \tilde{Q}=\left[\begin{array}{cc}
I & Y \\
0 & N^{T}
\end{array}\right],
$$




$$
\begin{aligned}
& {\left[\begin{array}{cccccc}
A \boldsymbol{X}+B \tilde{\boldsymbol{C}}+(\star) & { }^{\star} & \star & \star & \star & \star \\
\tilde{\boldsymbol{A}}+(A+B \hat{\boldsymbol{D}} C)^{T} & \boldsymbol{Y} A+\tilde{\boldsymbol{B}} \boldsymbol{y} C+(\star) & \star & \star & \star & \star \\
\left(B_{d}+B \hat{\boldsymbol{D}} D_{d}\right)^{T} & \left(\boldsymbol{Y} B_{d}+\tilde{\boldsymbol{B}} \boldsymbol{y} D_{d}\right)^{T} & -\gamma I & \star & \star & \star \\
\left(B_{f}+B \hat{\boldsymbol{D}} D_{f}\right)^{T} & \left(\boldsymbol{Y} B_{f}+\tilde{\boldsymbol{B}} \boldsymbol{y} D_{f}\right)^{T} & 0 & -\gamma I & \star & \star \\
C_{z} \boldsymbol{X}+D_{z} \tilde{\boldsymbol{C}} & C_{z}+D_{z} \hat{\boldsymbol{D}} C & D_{z d}+D_{z} \hat{\boldsymbol{D}} D_{d} & D_{z} \hat{\boldsymbol{D}} D_{f} & -\gamma I & \star \\
\tilde{\boldsymbol{C}} \boldsymbol{r} & \overline{\boldsymbol{D}}_{\boldsymbol{r} \boldsymbol{y} C} & \overline{\boldsymbol{D}}_{\boldsymbol{r} \boldsymbol{y} D_{d}} & \overline{\boldsymbol{D}} \boldsymbol{r y} D_{f}-I & 0 & -\gamma I
\end{array}\right]<0,} \\
& {\left[\begin{array}{cc}
X & I \\
I & Y
\end{array}\right]>0 \text {. }}
\end{aligned}
$$

from $P * P^{-1}=I$, we immediately have $P Q=\tilde{Q}$ and the following results:

$$
\begin{aligned}
& Q^{T} P A_{i n f} Q=\tilde{Q}^{T} A_{\text {inf }} Q=\left[\begin{array}{ll}
A_{11} & A_{12} \\
A_{21} & A_{22}
\end{array}\right], \\
& Q^{T} P B_{\text {inf }}=\tilde{Q}^{T} B_{\text {inf }}=\left[\begin{array}{ll}
B_{11} & B_{12} \\
B_{21} & B_{22}
\end{array}\right] \\
& C_{\text {inf }} Q=\left[\begin{array}{ll}
C_{11} & C_{12} \\
C_{21} & C_{22}
\end{array}\right]
\end{aligned}
$$

where

$$
\begin{aligned}
& A_{11}=A \boldsymbol{X}+B \hat{\boldsymbol{D}} C \boldsymbol{X}+B \hat{\boldsymbol{C}} M^{T}, \\
& A_{12}=A+B \hat{\boldsymbol{D}} C \text {, } \\
& A_{21}=\boldsymbol{Y} A \boldsymbol{X}+\boldsymbol{Y} B \hat{\boldsymbol{D}} C \boldsymbol{X}+N\left(\hat{\boldsymbol{B}}_{\boldsymbol{y}}+\hat{\boldsymbol{B}}_{\boldsymbol{u}} \hat{\boldsymbol{D}}\right) C \boldsymbol{X} \\
& +\boldsymbol{Y} B \hat{\boldsymbol{C}} M^{T}+N(\hat{\boldsymbol{A}}+\hat{\boldsymbol{B}} \boldsymbol{u} \hat{\boldsymbol{C}}) M^{T}, \\
& A_{22}=\boldsymbol{Y} A+\boldsymbol{Y} B \hat{\boldsymbol{D}} C+N(\hat{\boldsymbol{B}} \boldsymbol{y}+\hat{\boldsymbol{B}} \boldsymbol{u} \hat{\boldsymbol{D}}) C, \\
& B_{11}=B_{d}+B \hat{\boldsymbol{D}} D_{d} \\
& B_{12}=B_{f}+B \hat{\boldsymbol{D}} D_{f} \text {, } \\
& B_{21}=\boldsymbol{Y} B_{d}+\boldsymbol{Y} B \hat{\boldsymbol{D}} D_{d}+N(\hat{\boldsymbol{B}} \boldsymbol{y}+\hat{\boldsymbol{B}} \boldsymbol{u} \hat{\boldsymbol{D}}) D_{d}, \\
& B_{22}=\boldsymbol{Y} B_{f}+\boldsymbol{Y} B \hat{\boldsymbol{D}} D_{f}+N\left(\hat{\boldsymbol{B}}_{\boldsymbol{y}}+\hat{\boldsymbol{B}} \boldsymbol{u} \hat{\boldsymbol{D}}\right) D_{f} \text {, } \\
& C_{11}=C_{z} \boldsymbol{X}+D_{z} \hat{\boldsymbol{D}} C \boldsymbol{X}+D_{z} \hat{\boldsymbol{C}} M^{T} \text {, } \\
& C_{12}=C_{z}+D_{z} \hat{\boldsymbol{D}} C \\
& C_{21}=\left(\hat{\boldsymbol{D}}_{\boldsymbol{r} \boldsymbol{y}}+\hat{\boldsymbol{D}}_{\boldsymbol{r u}} \hat{\boldsymbol{D}}\right) C \boldsymbol{X}+\left(\hat{\boldsymbol{C}}_{\boldsymbol{r}}+\hat{\boldsymbol{D}}_{\boldsymbol{r u}} \hat{\boldsymbol{C}}\right) M^{T}, \\
& C_{22}=\left(\hat{\boldsymbol{D}}_{\boldsymbol{r y}}+\hat{\boldsymbol{D}}_{\boldsymbol{r u}} \hat{\boldsymbol{D}}\right) C \text {. }
\end{aligned}
$$

Then we can define

$$
\begin{aligned}
& \bar{A}=\hat{A}+\hat{B}_{u} \hat{C}, \\
& \overline{\boldsymbol{B}}_{\boldsymbol{y}}=\hat{\boldsymbol{B}}_{\boldsymbol{u}} \hat{\boldsymbol{D}}+\hat{\boldsymbol{B}}_{\boldsymbol{y}}, \\
& \bar{C}_{r}=\hat{C} \boldsymbol{r}+\hat{D}_{r u} \hat{C}, \\
& \bar{D}_{r y}=\hat{D}_{r y}+\hat{D}_{r u} \hat{D} \text {, } \\
& \tilde{\boldsymbol{A}}=\boldsymbol{Y} A \boldsymbol{X}+\boldsymbol{Y} B \hat{\boldsymbol{D}} C \boldsymbol{X} \\
& +N \overline{\boldsymbol{B}}_{\boldsymbol{y}} C \boldsymbol{X}+\boldsymbol{Y} B \hat{\boldsymbol{C}} M^{T}+N \overline{\boldsymbol{A}} M^{T}, \\
& \tilde{\boldsymbol{B}}_{\boldsymbol{y}}=\boldsymbol{Y} B \hat{\boldsymbol{D}}+N \overline{\boldsymbol{B}}_{\boldsymbol{y}}, \\
& \tilde{\boldsymbol{C}}=\hat{\boldsymbol{D}} C \boldsymbol{X}+\hat{\boldsymbol{C}} M^{T} \text {, } \\
& \tilde{\boldsymbol{C}}_{\boldsymbol{r}}=\overline{\boldsymbol{D}}_{\boldsymbol{r} \boldsymbol{y}} C \boldsymbol{X}+\overline{\boldsymbol{C}}_{\boldsymbol{r}} M^{T}
\end{aligned}
$$

If $M$ and $N$ are invertible, the variable $\overline{\boldsymbol{A}}, \overline{\boldsymbol{B}} \boldsymbol{y}, \hat{\boldsymbol{C}}, \overline{\boldsymbol{C}} \boldsymbol{r}$ can be replaced by the new variables $\tilde{\boldsymbol{A}}, \tilde{\boldsymbol{B}} \boldsymbol{y}, \tilde{\boldsymbol{C}}, \tilde{\boldsymbol{C}} \boldsymbol{r}$ without loss of generality. The constraint $P>0$, can be expressed as an
LMI as follows:

$$
P>0 \Leftrightarrow Q^{T} P Q>0 \Leftrightarrow\left[\begin{array}{cc}
\boldsymbol{X} & I \\
I & \boldsymbol{Y}
\end{array}\right]>0 .
$$

Also define $T=\operatorname{diag}(Q, I)$, then (11) is true if and only if $T^{T} T_{m i} T<0$, which results in (12) readily.

B. Exploitation of Redundant Controller Gains $\hat{B}_{u}$ and $\hat{D}_{r u}$

The following Lemma 3.2 shows that the controller gain $\hat{B}_{u}$ is redundant in the design procedure and can be removed from the GDC without loss of generality, where the choice of $\hat{B}_{u}$ will not affect optimality.

Lemma 3.2: Let all variables be defined as above and if a stabilizing dynamic controller such that $\left\|T_{i n f}\right\|_{\infty}<\gamma$ is achieved via Lemma 3.1 , then this controller with an arbitrary selection of $\hat{B}_{u}$ realises an identical controller for Problem 8.

Proof It is easy to see that

$$
A_{c l}=\left[\begin{array}{cc}
A+B \hat{D} C & B \hat{C} \\
\bar{B}_{y} C & \bar{A}
\end{array}\right]
$$

from our definitions in Lemma 3.1, where $\bar{B}_{y}$ and $\bar{A}$ comes directly from the numerical solutions of (12). It can also be seen from (12) that $\hat{B}_{u}$ does not even appear in the LMI iteration.

Therefore, the closed-loop and controller dynamics are not affected by the choice of $\hat{B}_{u}$.

Similarly, for $\hat{D}_{r u}$.

Referring to the above, we conclude that $\hat{B}_{u}$ and $\hat{D}_{r u}$ can be arbitrarily chosen without affecting the optimality achieved in the LMI solution.

\section{ILLUSTRATIVE EXAMPLE}

In this section a rather practical example is utilised to illustrate the applicability of the proposed approach of combined control, fault tolerance and fault diagnosis in dynamics systems. The problem addressed is ride quality maintenace of high speed railway vehicles. The mathematical model of system is based on the side-view of a railway vehicle as illustrated in Figure 2, considering both the bounce and pitch motions of the vehicle body and only the bounce motion of the bogie masses. The suspensions, which include the primary suspensions and secondary suspensions, are represented by dampers and springs in parallel. In fact, the primary suspension is mainly for providing guidance of the vehicle and the secondary suspension is aimed to improve 
the ride quality of the vehicle. Active control is provided by actuators placed across the front and rear secondary suspensions. The control objective is to achieve good ride quality while maintaining adequate suspension clearance, i.e. minimizing the acceleration of the vehicle body experienced by passengers without causing large suspension deflections. The dynamics of the model is given by [12]

$$
\dot{\mathbf{x}}=\mathbf{A x}+\mathbf{B}_{\mathbf{d}} \mathbf{d}+\mathbf{B u}
$$

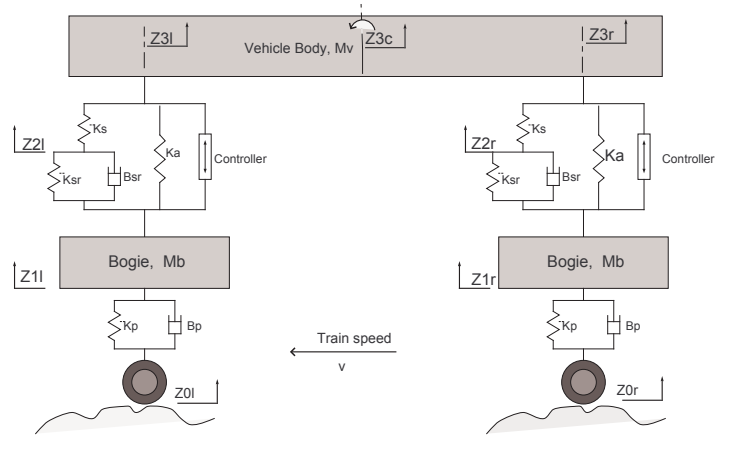

Fig. 2. A suspension system of a railway vehicle

We consider only the rigid motion of the railway vehicle body, where the set of states selected in the state space model are translational velocities of the three masses, the rotational velocity of the vehicle body, and deflections across the various springs, i.e.

$$
\begin{aligned}
& x=\left[\begin{array}{lllllll}
\dot{Z}_{3 c} & \dot{\theta} & \dot{Z}_{1 l} & \dot{Z}_{1 r} & Z_{3 l}-Z_{1 l} & Z_{3 r}-Z_{1 r} & Z_{2 l}-Z_{1 l}
\end{array}\right. \\
& \left.Z_{2 r}-Z_{1 r} \quad Z_{1 l}-Z_{0 l} \quad Z_{1 r}-Z_{0 r}\right]
\end{aligned}
$$

The suspension control inputs (active forces provided by actuator systems between the bogies and the body) and track disturbance inputs are given by

$$
\mathbf{u}=\left[\begin{array}{ll}
U_{l} & U_{r}
\end{array}\right], \mathbf{d}=\left[\begin{array}{ll}
\dot{Z}_{0 l} & \dot{Z}_{0 r}
\end{array}\right] .
$$

The corresponding state space system matrices are provided as follows. Since the control objective is to maintain ride quality via minimizing the acceleration of the vehicle body, the regulated outputs are chosen to characterise bounce accelerations of the vehicle body, i.e., $\ddot{Z}_{3 c}, \ddot{Z}_{3 l}, \ddot{Z}_{3 r}$. The following sensor information is available:

1) bounce acceleration sensor at left to measure $\ddot{Z}_{3 l}$

2) bounce acceleration sensor at right to measure $\ddot{Z}_{3 r}$

3) deflection sensor to measure $Z_{l}=Z_{3 l}-Z_{1 l}$

4) deflection sensor to measure $Z_{r}=Z_{3 r}-Z_{1 r}$

While, the output equation with full measurements is given by

$$
\mathbf{y}=\mathbf{C x}+\mathbf{D}_{\mathbf{d}} \mathbf{d}+\mathbf{D u}
$$

where

$$
C=\left[\begin{array}{cccccccccc}
0 & 0 & 0 & 0 & -66.43 & 12.96 & 66.43 & -12.96 & 0 & 0 \\
0 & 0 & 0 & 0 & 12.96 & -66.43 & -12.96 & 66.43 & 0 & 0 \\
0 & 0 & 0 & 0 & 1 & 0 & 0 & 0 & 0 & 0 \\
0 & 0 & 0 & 0 & 0 & 1 & 0 & 0 & 0 & 0
\end{array}\right],
$$

$$
D=(1.0 e-004) *\left[\begin{array}{cc}
-0.65 & 0.13 \\
0.13 & -0.65 \\
0 & 0 \\
0 & 0
\end{array}\right], D_{d}=0
$$

Note that $D \neq 0$, however this can be modified in order to apply our algorithm directly via loopshifting [13]. We choose all distribution matrices for regulated signals from the output equation. In the remaining of this paper, case studies are carried out based on the selection of above measurements and potential source of sensor faults. It is worth noting that we illustrate the proposed framework with emphasizing strictly the best ride quality control strategy (for the latter please refer to [12] and references within).

With full measurements, we consider two faults occurring in the left and right deflection sensors with distribution matrices given by

$$
B_{f}=0, D_{f}=\left[\begin{array}{llll}
0 & 0 & 1 & 0 \\
0 & 0 & 0 & 1
\end{array}\right]^{T} .
$$

Note that this is no strict condition and other fault scenaria can be freely chosen. In addition, the control design objective is chosen as to ensure a worst case control performance with passive fault tolerance and fault diagnosis via a generic $\mathcal{H}_{\infty}$ dynamic controller, with the presence of faults. Lemma 3.1 gives a generic $\mathcal{H}_{\infty}$ dynamic controller and an optimal $\gamma_{0}=$ 0.2155 .

A time-domain simulation result is also given to verify that the controller design does not significantly lose acceptable control performance when faults occur while maintaining appropriate disturbance attenuation. Figure 3 shows the time response with the full set subject to left deflection sensor fault $f_{3}$ and right deflection sensor fault $f_{4}$, where $f_{3}$ is simulated by an abrupt jump from the $2 n d$ second and $f_{4}$ is a negative unit step from the $6 t h$ second. Moreover, both track disturbances (i.e. the original and delayed versions) are Gaussian noises with mean zero and variance is $2 \pi^{2} A r \times v$ (one-sided) for a speed of $v=55(\mathrm{~m} / \mathrm{s})$ and a typical good quality track with track roughness $A r=2.5 e-7(m)$. Meanwhile, the residual signal from this generic dynamic controller can perfectly indicate occurring faults, as shown in Figure 4.

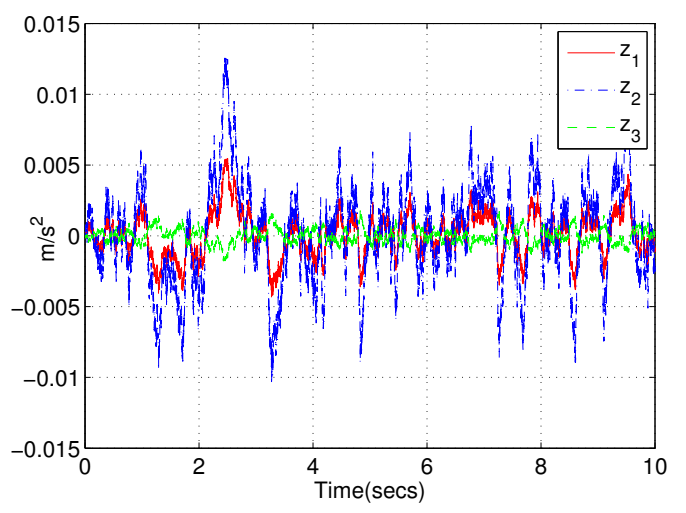

Fig. 3. Time response for regulated outputs with faults $f_{3}, f_{4}$. 


$$
\begin{aligned}
& A=\left[\begin{array}{cccccccccc}
0 & 0 & 0 & 0 & -26.7368 & -26.7368 & 26.7368 & 26.7368 & 0 & 0 \\
0 & 0 & 0 & 0 & 4.1784 & -4.1784 & -4.1784 & 4.1784 & 0 & 0 \\
0 & 0 & -40.592 & 0 & 406.4 & 0 & -406.4 & 0 & -3948 & 0 \\
0 & 0 & 0 & -40.592 & 0 & 406.4 & 0 & -406.4 & 0 & -3948 \\
1 & -9.5 & -1 & 0 & 0 & 0 & 0 & 0 & 0 & 0 \\
1 & 9.5 & 0 & -1 & 0 & 0 & 0 & 0 & 0 & 0 \\
0 & 0 & 0 & 0 & 15.8478 & 0 & -23.7716 & 0 & 0 & 0 \\
0 & 0 & 0 & 0 & 0 & 15.8478 & 0 & -23.7716 & 0 & 0 \\
0 & 0 & 1 & 0 & 0 & 0 & 0 & 0 & 0 & 0 \\
0 & 0 & 0 & 1 & 0 & 0 & 0 & 0 & 0 & 0
\end{array}\right], \\
& B=0.001 *\left[\begin{array}{cc}
-0.0263 & -0.0263 \\
0.0041 & -0.0041 \\
0.4000 & 0 \\
0 & 0.4000 \\
0 & 0 \\
0 & 0 \\
0 & 0 \\
0 & 0 \\
0 & 0 \\
0 & 0
\end{array}\right], B_{d}=\left[\begin{array}{cc}
0 & 0 \\
0 & 0 \\
40.5920 & 0 \\
0 & 40.5920 \\
0 & 0 \\
0 & 0 \\
0 & 0 \\
0 & 0 \\
-1.0000 & 0 \\
0 & -1.0000
\end{array}\right]
\end{aligned}
$$

\section{ACKNOWLEDGMENTS}

This research was supported under the EPSRC project Grant Ref. EP/D063965/1 and BAE Systems.

\section{REFERENCES}

[1] X. Ding and P. Frank, "Fault detection via factorization approach," Systems \& Control Letts., vol. 14, pp. 431-436, 1990.

[2] P. M. Frank, "Fault diagnosis in dynamic systems using analytical and knowledge-based redundancy: A survey and some new results," Automatica, vol. 26, no. 3, pp. 459-474, 1990.

[3] P. M. Frank and X. Ding, "Survey of robust residual generation and evaluation methods in observer-based fault detection systems," J. Proc. Cont., vol. 7, no. 6, pp. 403-424, 1997.

[4] R. Patton, P. Frank, and R. C. (Eds), Issues of Fault Diagnosis for Dynamic Systems. Springer, 2000.

[5] J. Stoustrup, M. J. Grimble, and H. Niemann, "Design of integrated systems for the control and detection of actuator/sensor faults," Sensor Review, vol. 17, no. 2, pp. 138-149, 1997.

[6] H. Niemann and J. Stoustrup, "Passive fault tolerant control of a double inverted pendulum-a case study," Control Engineering Practice, vol. 13, pp. 1047-59, 2005.

[7] Z. Li, A. C. Zolotas, I. M. Jaimoukha, and K. M. Grigoriadis, "Output selection with fault tolerance via dynamic controller design," in Proceedings of the 17th Inernational Federation of Automatic Control World Congress, Seoul, Korea, July 2008, pp. 2570-2575.

[8] I. Jaimoukha, Z. Li, and V. Papakos, "A matrix factorization solution to the $\mathcal{H}_{-} / \mathcal{H}_{\infty}$ fault detection problem," Automatica, vol. 42, no. 11, pp. 1907-1913, 2006.

[9] R. J. Patton and J. Chen, "Observer-based fault detection and isolation: Robustness and applications," Control Engineering Practice, vol. 5, no. 5, pp. 671-682, 1997.

[10] C. Scherer, P. Gahinet, and M. Chilali, "Multi-objective outputfeedback control via LMI optimization," IEEE Trans. Automatic Control, vol. 42, no. 7, pp. 896-911, 1997.

[11] S. Boyd, L. El Ghaoui, E. Feron, and V. Balakrishnan, Linear Matrix Inequalities in Systems and Control Theory. Philadelphia, PA: SIAM Publications, 1994.

[12] X. Zheng, A. C. Zolotas, and R. M. Goodall, "Modeling of flexiblebodied railway vehicles for vibration suppression," in ICSE, Coventry, UK, 2006.

[13] M. Safonov and D. Limebeer, "Simplifying the $H^{\infty}$ theory via loop shifting," in Proc. IEEE Conf. Dec. \& Control, Austin, TX, Dec. 1988, IEEE Press, New York. 